INTERNATIONAL DICTIONARY OF THE SECURITIES INDUSTRY 


\title{
INTERNATIONAL DICTIONARY OF THE
}

\section{SECURITIES INDUSTRY}

\author{
Stuart Valentine
}

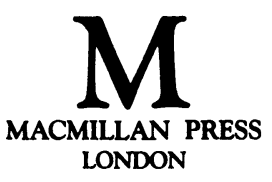

Macmillan Reference Books 
All rights reserved. No reproduction, copy or transmission of this publication may be made without permission.

No paragraph of this publication may be reproduced, copied or transmitted save with written permission or in accordance with the provisions of the Copyright Act 1956 (as amended). Any person who does any unauthorised act in relation to this publication may be liable to criminal prosecution and civil claims for damages.

First published 1985 by

THE MACMILLAN PRESS LTD

London and Basingstoke

Associated companies in Auckland, Delhi, Dublin, Gaborone, Hamburg, Harare, Hong Kong, Johannesburg, Kuala Lumpur, Lagos, Manzini, Melbourne, Mexico City, Nairobi, New York, Singapore, Tokyo.

\title{
British Library Cataloguing in Publication Data
}

\author{
Valentine, S.P. \\ International dictionary of securities industry. \\ 1. Securities \\ I. Title \\ 332.65 HG4528
}

ISBN 978-0-333-38241-7 ISBN 978-1-349-17889-6 (eBook)

DOI $10.1007 / 978-1-349-17889-6$

The paperback edition of this book is sold subject to the condition that is shall not, by way of trade or otherwise, be lent, resold, hired out, or otherwise circulated without the publisher's prior consent in any form of binding or cover other than that in which it is published and without a similar condition including this condition being imposed on the subsequent purchaser. 


\section{INTRODUCTION}

It has long been one of my hobby-horses that the essential workings of a stock market are really quite simple. Yet new investors approaching the market for the first time are apt to be thoroughly put off by the apparent complexity of the language. That is a pity, because little can be more important for someone than to use his savings for his own and his family's security. Not understanding the world of investment and blindly trusting those who use the language as a smoke-screen, rather than as the means of communication it is supposed to be, can have unfortunate consequences: at best the saver will not use his savings to their best advantage, while at worst, he could be lured into highly speculative investments by the promise of rich rewards, without appreciating the risks involved.

The language can be off-putting: indeed, the whole investment scene can sound at times like a safari park, with its inhabitants of bulls, bears, stags, cats and tigers, a veritable thundering herd of wildlife. Yet all of these have their meanings in everyday language and all of them (including the thundering herd itself) will be found in the pages of this volume.

The object of compiling this book was not, of course, to solve all financial problems and make everyone rich. Anyone looking for such a book is advised to put down this volume right now and try another. Rather, its aim is to help both those approaching investment for the first time and also those already involved to thread their way through the jargon. Those involved will already know better than I the meanings of many of the words here. But the language of investment is growing week by week, and I hope that even the experienced investor and market man will find something new here.

The world of investment is also growing more international. Phrases starting in one market gradually (or these days not so gradually) pass into other markets' terminology. As the market in securities is becoming increasingly worldwide, an attempt has been made here to explain some of the words and the institutions of overseas markets, but I am conscious that many words common in those markets may have been left out.

The other problem is the rate of growth, amounting almost to an explosion, in the language of investment. Dozens of the words and phrases in this dictionary would not have been there a year ago. Frighteningly, a year from now there will be dozens more which today are still unheard of.

So, inevitably, not everything will be here, but I hope that it will meet the needs of those who, in their own or in overseas markets, are in need of clarification and enlightenment on the meaning of particular words and phrases. Where possible I have tried to include sources of further information, by way of bibliography, in the hope that those who find the definition given here inadequate for their needs will be guided to the more detailed information in those other sources.

I should say a word or two about layout and style. A strict alphabetical order has been adopted, ignoring the fact that individual words may be separated: thus 'capitalization' comes after 'capital gain' but before 'capital redemption yield'.

Cross references to both English and foreign words contained in the body of the text are printed in SMALL CAPITALS. For foreign terminology, where the word or phrase has a close English equivalent, I have referred readers to that English translation although, occasionally, the cross-referenced English equivalent will be accompanied by background information which refers to the specific situation in the United Kingdom or the United States. Where there is no such equivalent, an explanation of the phrase has, of course, been given. 


\section{vi Introduction}

The words bourse, Börse and Borsa have been used in the appropriate national contexts, although I have sometimes used phrases such as 'the French Stock Exchanges' or 'the Frankfurt Stock Exchange' when to have used bourse or Börse would have sounded excessively pedantic.

The differences between English and American usage have sometimes caused difficulty. Though 'company' and 'corporation' may be used almost interchangeably, I have tried to be careful to distinguish between the English and American uses of the word 'stock': in the United Kingdom 'stock' is a fixed interest security; in the United States, 'stock' or 'common stock' is equity, the word for fixed interest security being 'bond'. If it is any consolation to the layman, conversations between market practitioners of the two countries often have to be clarified on this score. In any discrepancy between English and American spelling the English version has been used except where the definition in which the word appears is wholly American. The style, -ize and -ization have been used at the publisher's request, instead of -ise and -isation.

Where a word has (a) a common meaning; (b) a banking or other financial meaning; (c) a specific stock exchange meaning, I have tended to ignore the first of these, and have defined the second only when it has a relevance to a stock market, or is necessary to understand the particular use employed in a stock exchange context. Thus, for example, I have not defined the word 'average' in an insurance context, only its stock market meaning. In all cases, the specific stock exchange meaning has been defined.

Finally, I have used the word 'he' to refer to the third person singular throughout. 


\section{ACKNOWLEDGEMENTS}

Anyone attempting to compile a dictionary which sets out to cover such a broad spectrum as international stock market terminology must, of necessity, rely on the active and enthusiastic assistance of others. In this case, that help has been so unstintingly given by so many people that to do them all full justice would fill far more pages than I am allotted for the purpose.

In particular, I would like to thank first and foremost Malcolm Duncan of the Milan Stock Exchange for the immense amount of help willingly given on Italian terminology. Also Bill Freund and Kenneth Fox of the New York Stock Exchange for the use of the NYSE glossary of terminology, as well as for considerable help given me in clarifying and supplementing my information on the complex American scene. In this context, my additional thanks are due to Merril Stevenson of The Economist for invaluable help and advice.

On other overseas markets I have been greatly assisted by the help I have received from Bertrand Jacquillat and Bruno Montier of the Chambre Syndicale des Agents de Change in Paris; by Dr. Herbert Schlicht of the Frankfurt Stock Exchange, Robert Fell, Commissioner for Securities in Hong Kong, Ada Jeung of his office and Andrew Mason of the London office of Nomura Research.

I should like to thank Dr Paul Stonham for permission to use material from his book, Major Stock Markets of Europe, references to which appear in various bibliographies in the text. John Wiley, the New York publishers, also gave me permission to quote from their International Finance Handbook, edited by Abraham M. George and Ian H. Giddy, and again the debt I owe to that volume is reflected in many of the bibliographic notes. So too is the debt to Janette Rutterford's book, An Introduction to Stock Exchange Investment.

On the UK domestic scene, Juliette Proudlove of the London International Financial Futures Exchange provided me with valuable information on that market's terminology, while Tony Newman and A.E. Backholer, of the Society of Investment Analysts, kindly provided help on balance sheet terminology and on the sometimes complex vocabulary of the investment analyst. Among my colleagues at The Stock Exchange I would like to thank Sheila Nicoll for providing many valuable contacts and for help with translations, together with Alan King and Tracy Alexander of the Settlement Division, who helped guide me through some of the more obscure corners of that esoteric area.

To all of those mentioned, as well as many who gave pieces of advice too numerous to mention, I extend my most sincere thanks.

Their contribution is a major one, but I must not forget those who gave me encouragement, support and help in the actual production of the volume: Jeffrey Knight, Chief Executive of The Stock Exchange, who encouraged me to undertake the task; David Clutterbuck and John Hodgson of Macmillan Press who initiated the idea and helped me to carry it to fruition; John Webster, whose technical expertise helped speed up the production process; Nigel Storer, who helped check information and fill in some of the blanks. My thanks most of all go to Ann Hanson, who not only typed and re-typed the drafts, but who was also extremely valuable in making me turn highly obscure and unintelligible definitions into something approaching both clarity and the English language. Obscurities and errors which remain are entirely, of course, my own responsibility.

A final word of gratitude to my wife Jill, whose forebearance when the evenings and weekends of what was laughingly called my spare time were devoted to 'The Dictionary'. When I emerge from the study, I only hope she remembers who I am.

To all those who have given their help, I am aware I have built up a large debit balance of favours owed. I hope I shall have the opportunity to repay them. 


\section{List of Abbreviations}

The following commonly-used abbreviations and acronyms appear in the main body of the text in the alphabetical order of the full reference. Where an abbreviation or acronym has itself been used as a headword, e.g. TALISMAN, it has not been repeated here.

\begin{tabular}{|c|c|c|c|}
\hline AASE & $\begin{array}{l}\text { : Association of Australian } \\
\text { Stock Exchanges }\end{array}$ & $\begin{array}{l}\text { CGT } \\
\text { CMA }\end{array}$ & $\begin{array}{l}\text { : Capital Gains Tax } \\
\text { : cash management account }\end{array}$ \\
\hline ABS & : Automated Bond System & $\mathrm{COB}$ & : Commission des Opérations \\
\hline ACT & : Advance Corporation Tax & & de Bourse \\
\hline ADR & : American Depository Receipt & CONSOB & : Commissione Nazionale per \\
\hline $\mathrm{AG}$ & : Aktiengesellschaft & & la Società e la Borsa \\
\hline $\mathrm{AHC}$ & : Accepting Houses Committee & CSI & : Council for the Securities \\
\hline AIBD & $\begin{array}{l}\text { Association of International } \\
\text { Bond Dealers }\end{array}$ & CTT & $\begin{array}{l}\text { Industry } \\
\text { : Capital Transfer Tax }\end{array}$ \\
\hline AITC & : Association of Investment & & \\
\hline AMEX & $\begin{array}{l}\text { Trust Companies } \\
\text { : American Stock Exchange }\end{array}$ & DOTS & $\begin{array}{l}\text { : Designated Order Turnaround } \\
\text { System }\end{array}$ \\
\hline AMOS & $\begin{array}{l}\text { : AMEX Options Switching } \\
\text { System }\end{array}$ & DTC & : Depository Trust Company \\
\hline $\begin{array}{l}\text { ARP } \\
\text { ASAS }\end{array}$ & $\begin{array}{l}\text { : adjustable rate preferred stock } \\
\text { : American Shares Amsterdam }\end{array}$ & DTI & $\begin{array}{l}\text { Department of Trade and } \\
\text { Industry }\end{array}$ \\
\hline ASL & $\begin{array}{l}\text { System } \\
\text { Admission of Securities to } \\
\text { Listing }\end{array}$ & $\begin{array}{l}\text { ECI } \\
\text { EDSP }\end{array}$ & $\begin{array}{l}\text { : Equity Capital for Industry } \\
\text { : exchange delivery settlement } \\
\text { price }\end{array}$ \\
\hline BES & : Business Expansion Scheme & EFP & : exchange for physicals \\
\hline BET & book entry transfer & $\begin{array}{l}\text { EMH } \\
\text { EOE }\end{array}$ & $\begin{array}{l}\text { : Efficient Market Hypothesis } \\
\text { : European Options Exchange }\end{array}$ \\
\hline $\mathrm{CAC}$ & $\begin{array}{l}\text { : Compagnie des Agents de } \\
\text { Change }\end{array}$ & EPIC & $\begin{array}{l}\text { Electronic Price Information } \\
\text { Computer }\end{array}$ \\
\hline CAD & : cash against documents & EPS & : earnings per share \\
\hline CAES & $\begin{array}{l}\text { Computer Assisted Execution } \\
\text { System }\end{array}$ & ERISA & $\begin{array}{l}\text { Employee Retirement Income } \\
\text { Security Act }\end{array}$ \\
\hline CAP & $\begin{array}{l}\text { convertible adjustable } \\
\text { preferred stock }\end{array}$ & ESOP & $\begin{array}{l}\text { : Employee Stock Ownership } \\
\text { Plan }\end{array}$ \\
\hline CAPM & : Capital Asset Pricing Model & & \\
\hline CATS & $\begin{array}{l}\text { : Computer Assisted Trading } \\
\text { System (Canada) }\end{array}$ & FCI & $\begin{array}{l}\text { : Finance Corporation for } \\
\text { Industry }\end{array}$ \\
\hline CATS & $\begin{array}{l}\text { certificate of accrual on } \\
\text { Treasury securities (US) }\end{array}$ & $\begin{array}{l}\text { FESE } \\
\text { FFI }\end{array}$ & $\begin{array}{l}\text { : Far Eastern Stock Exchange } \\
\text { : Finance for Industry }\end{array}$ \\
\hline CBOE & $\begin{array}{l}\text { Chicago Board Options } \\
\text { Exchange }\end{array}$ & FHLMA & $\begin{array}{l}\text { Federal Home Loan } \\
\text { Mortgage Association }\end{array}$ \\
\hline CD & : certificate of deposit & FIBV & : Fédération Internationale des \\
\hline CFT & $\begin{array}{l}\text { : Commodity Futures Trading } \\
\text { Cornoration }\end{array}$ & IFO & $\begin{array}{l}\text { Bourses de Valeurs } \\
\text { first-in-first-out }\end{array}$ \\
\hline
\end{tabular}




\begin{tabular}{|c|c|}
\hline FNMA & $\begin{array}{l}\text { : Federal National Mortgage } \\
\text { Association }\end{array}$ \\
\hline FOCUS & $\begin{array}{l}\text { Financial and Operational } \\
\text { Combined Uniform Single } \\
\text { Report }\end{array}$ \\
\hline FTA & $\begin{array}{l}\text { : Financial Times-Actuaries all } \\
\text { share index }\end{array}$ \\
\hline FTO & $\begin{array}{l}\text { : Financial Times Ordinary } \\
\text { share index }\end{array}$ \\
\hline FTSE & $\begin{array}{l}\text { : Financial Times-Stock } \\
\text { Exchange index }\end{array}$ \\
\hline
\end{tabular}

GmbH : Gesellschaft mit beschränkter Haftung (see Gesellschaft)

GNMA : Government National Mortgage Association

$\begin{array}{ll}\text { HSI } & : \text { Hang Seng Index } \\ \text { IBRD } & : \text { International Bank for } \\ & \text { Reconstruction and } \\ & \text { Development }\end{array}$

ICCH : International Commodities Clearing House

ICFC : Industrial and Commercial Finance Corporation
ID : International Dealer
IFC : International Finance Corporation

IHA : Issuing Houses Association

III : Investors In Industry

IMM : International Monetary Market

IRA : Individual Retirement Account

ITS : Intermarket Trading System

KAGG : Gesetz über die Kapitalanlagegesellschaften

KNI : Kam Ngan Index

LCM : Limited Corporate Member

LDMA : London Discount Market Association

LIBOR : London Inter-Bank Offered Rate

LIFFE : London International Financial Futures Exchange

LIFO : last-in-first-out

LSE : London Stock Exchange
MIB : indice Borsa Valori di Milano

MIT : market-if-touched

MLR : Minimum Lending Rate

MPDS : Market Price Display Service
NASD : National Association of Securities Dealers (US)
NASDAQ : National Association of Security Dealers Automated Quotations System
NASDIM : National Asociation of Securities Dealers and Investment Managers (UK)
NDJA : Nikkei-Dow Jones Average
NPV : no par value (shares)
NSCC : National Securities Clearing Corporation

NYFE : New York Futures Exchange

NYSE : New York Stock Exchange

OARS : Opening Automated Report Service

OFT : Office of Fair Trading

OMO : open market operations

OTC : over-the-counter (markets)

PE or PER : price-earnings ratio

PLC : Public Limited Company

PSL (1 or 2) : private sector liquidity

R4 : Registered Representative Rapid Response System

REIT : real estate investment trust

RPI : retail price index

SA : société anonyme

SAYE : Save-As-You-Earn

SEC : Securities and Exchange

Commission

SEDOL : Stock Exchange Daily Official List

SEPON : Stock Exchange Pool Nominees

SESI : Stock Exchange of Singapore Index

SEOYB : Stock Exchange Official Year Book

SIA : Securities Industry Association

SIAC : Securities Industry Automation Corporation 
x Abbreviations

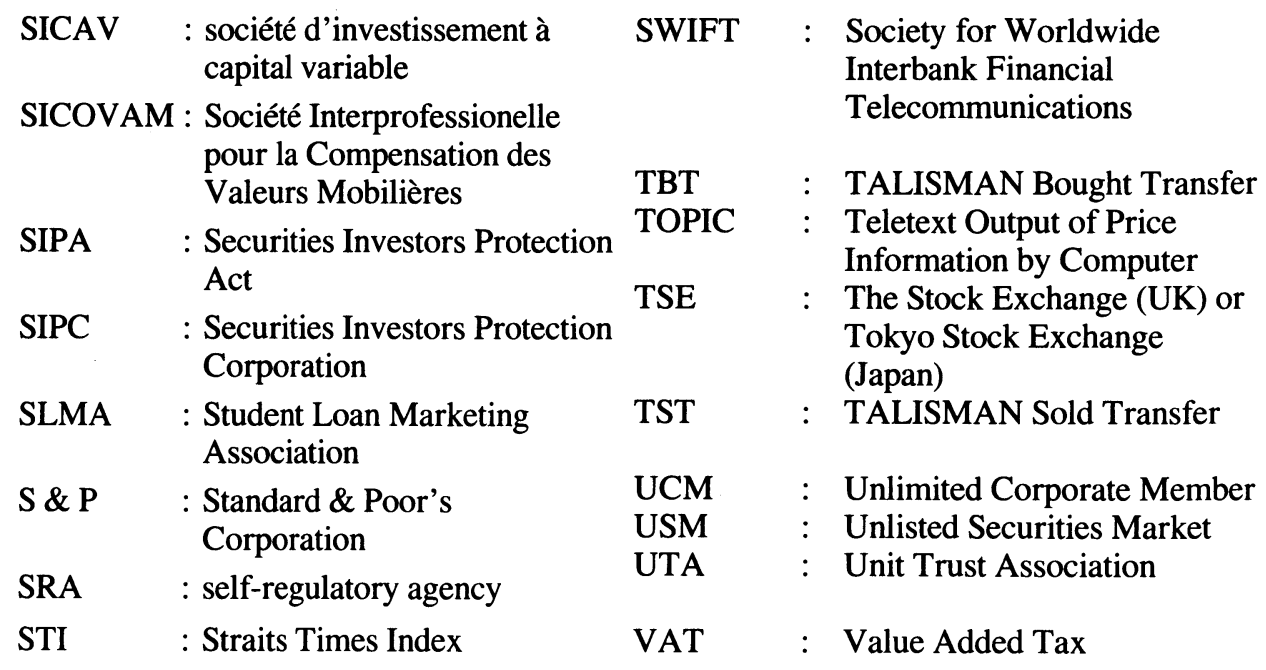




\section{World Stock Exchange Indices}

\begin{tabular}{|c|c|c|c|c|c|}
\hline Country & Index & $\begin{array}{l}\text { No. of } \\
\text { Companies }\end{array}$ & Base date & $\begin{array}{l}\text { Base } \\
\text { Value }\end{array}$ & $\begin{array}{l}\text { Frequency of } \\
\text { Calculation }\end{array}$ \\
\hline Argentina & $\begin{array}{l}\text { El Cronista Commercial-Banco } \\
\text { Roberts }\end{array}$ & All (260 app.) & 29 Dec 1977 & 100 & daily \\
\hline \multirow[t]{3}{*}{ Australia } & $\begin{array}{l}\text { Australian Stock Exchange All } \\
\text { Ordinaries Price Index (AOPI) }\end{array}$ & 270 (app.) & 31 Dec 1979 & 500 & continuously \\
\hline & $\begin{array}{l}\text { Australian Stock Exchange All } \\
\text { Industrial Price Index (AIPI) }\end{array}$ & 80 (app.) & 31 Dec 1979 & 500 & continuously \\
\hline & $\begin{array}{l}\text { Australian Stock Exchange All } \\
\text { Resources Price Index (ARPI) }\end{array}$ & 190 (app.) & 31 Dec 1979 & 500 & continuously \\
\hline \multirow[t]{3}{*}{ Austria } & Creditanstalt Bankverein (CA Index) & 20 & 2 Jan 1962 & 100 & daily \\
\hline & $\begin{array}{l}\text { Girozentrale und Bank der } \\
\text { Osterreichischen Sparkassen AG } \\
\text { (GZ-Index) }\end{array}$ & All & 31 Dec 1966 & 100 & daily \\
\hline & Borsekammer (WBK Index) & 43 & 31 Dec 1967 & 100 & twice monthly \\
\hline \multirow[t]{2}{*}{ Belgium } & Cash Market Index & All (197) & 1 Jan 1980 & 100 & daily \\
\hline & $\begin{array}{r}\text { Account Market Index: foreign comp } \\
\text { Belgian comp }\end{array}$ & $\begin{array}{l}\text { nies All (63) } \\
\text { nies All (33) }\end{array}$ & $\begin{array}{l}1 \text { Jan } 1985 \\
1 \text { Jan } 1980\end{array}$ & $\begin{array}{l}1000 \\
1000\end{array}$ & $\begin{array}{l}\text { every two minutes } \\
\text { during trading } \\
\text { session }\end{array}$ \\
\hline Brazil & The Profit Index (IBV) & 34 & 16 Aug 1965 & 100 & daily \\
\hline \multirow[t]{3}{*}{ Canada } & Montreal Composite Index & 85 & 1956 & 100 & half-hourly \\
\hline & Industrial Index & 65 & 1956 & 100 & half-hourly \\
\hline & $\begin{array}{l}\text { Toronto " } 300 \text { "' composite stock } \\
\text { price index }\end{array}$ & 300 & 1975 & 1000 & $\begin{array}{l}\text { every } 15 \\
\text { minutes }\end{array}$ \\
\hline Denmark & Copenhagen Stock Exchange Index & 38 & 1 Jan 1973 & 100 & daily \\
\hline \multirow[t]{5}{*}{ France } & CAC Index & 268 & 31 Dec 1981 & 100 & at opening \\
\hline & CAC Trend Indicator & 50 & $\begin{array}{l}\text { end of } \\
\text { previous year }\end{array}$ & 100 & continuously \\
\hline & INSEE daily & 50 & $\begin{array}{l}\text { end of } \\
\text { previous year }\end{array}$ & 100 & at close \\
\hline & INSEE weekly & 220 & end 1981 & 100 & at opening \\
\hline & Agefi (cash and account market) & 162 & 2 Jan 1962 & 100 & at close \\
\hline Germany & Commerzbank & $\begin{array}{l}60 \text { "blue } \\
\text { chip" } \\
\text { companies }\end{array}$ & 1 Dec 1953 & 100 & daily \\
\hline Hong Kong & $\begin{array}{l}\text { Hang Seng Index } \\
\text { Far East Index }\end{array}$ & $\begin{array}{l}33 \\
52\end{array}$ & $\begin{array}{l}31 \text { July } 1964 \\
1 \text { Apr } 1971\end{array}$ & $\begin{array}{r}100 \\
1000\end{array}$ & \\
\hline Israel & $\begin{array}{l}\text { Total Yields indices for stocks } \\
\text { and bonds (TY) }\end{array}$ & all & 31 Dec 1981 & 100 & daily \\
\hline \multirow[t]{2}{*}{ Italy } & $\begin{array}{l}\text { Milano Indice borsa (MIB) } \\
\text { Current Index }\end{array}$ & 176 & 2 Jan 1983 & 1000 & daily \\
\hline & Historical Index & 129 & 2 Jan 1975 & 1000 & daily \\
\hline
\end{tabular}


xii World Stock Exchange Indices

\begin{tabular}{|c|c|c|c|c|c|}
\hline Country & Index & $\begin{array}{l}\text { No. of } \\
\text { Companies }\end{array}$ & Base date & $\begin{array}{l}\text { Base } \\
\text { Value }\end{array}$ & $\begin{array}{l}\text { Frequency of } \\
\text { Calculation }\end{array}$ \\
\hline \multirow[t]{2}{*}{$\begin{array}{l}\text { Japan } \\
\text { Osaka }\end{array}$} & Stock prices average (1st section) & 220 & 4 Jan 1968 & 100 & $\begin{array}{l}6 \text { times on full } \\
\text { business day }\end{array}$ \\
\hline & Stock prices average (2nd section) & 80 & 4 Jan 1968 & 100 & $\begin{array}{l}3 \text { times on half } \\
\text { business day }\end{array}$ \\
\hline \multirow[t]{2}{*}{ Tokyo } & $\begin{array}{l}\text { Toyko Stock Eschange Stock } \\
\text { prices indices (TSESPI) }\end{array}$ & $\begin{array}{l}\text { all on } 1 \text { st } \\
\text { section }\end{array}$ & 4 Jan 1968 & 100 & $\begin{array}{l}\text { 1st and 2nd } \\
\text { sections } \\
\text { continuously }\end{array}$ \\
\hline & Nikkei Dow Jones average & $\begin{array}{l}225 \text { on } \\
\text { 1st section }\end{array}$ & 16 May 1949 & 100 & \\
\hline Korea & $\begin{array}{l}\text { Korean Composite Stock price } \\
\text { Index (KCSPI) }\end{array}$ & all & 4 Jan 1980 & 100 & half-hourly \\
\hline Luxembourg & Luxembourg Shares Index & 8 & 27 Dec 1967 & 100 & daily at close \\
\hline Malaysia & $\begin{array}{l}\text { Kuala Lumpur Stock Exchange } \\
\text { Index (KLSE) }\end{array}$ & 30 & 1970 & 100 & $\begin{array}{l}\text { daily at close } \\
\text { and during } \\
\text { every recess }\end{array}$ \\
\hline Netherlands & ANP-CBS Stock Market Index & 53 & 1970 & 100 & $\begin{array}{l}\text { active } 4 \text { times } \\
\text { daily less active } \\
\text { twice daily }\end{array}$ \\
\hline Norway & Oslo SE Composite & 25 & 1 Jan 1972 & 100 & daily at close \\
\hline \multirow[t]{3}{*}{ Singapore } & $\begin{array}{l}\text { Stock Exchange of Singapore (SES) } \\
\text { Industrial \& Commercial }\end{array}$ & 32 & 31 Dec 1973 & 100 & twice daily \\
\hline & Straits Times industrial (ST) & 30 & 30 Dec 1966 & 100 & daily at close \\
\hline & OCBC Indices & 40 & 2 Jan 1970 & 100 & daily at close \\
\hline \multirow[t]{2}{*}{ South Africa } & $\begin{array}{l}\text { Johannesburg Stock Exchange } \\
\text { actuaries index (JSEA) }\end{array}$ & 146 & 31 Dec 1959 & 100 & continuously \\
\hline & Rand Daily Mail Indices & 100 & 1958 & 100 & daily at close \\
\hline $\begin{array}{l}\text { Spain: } \\
\text { Barcelona }\end{array}$ & $\begin{array}{l}\text { The Barcelona Stock Exchange } \\
\text { current and historical indices }\end{array}$ & 89 & $\begin{array}{l}1 \text { Jan each } \\
\text { year and } 1 \text { Jan } \\
1963 \text { for long } \\
\text { base index }\end{array}$ & 100 & continuously \\
\hline Madrid & Daily Index & 69 & $\begin{array}{l}31 \text { Dec of } \\
\text { previous year }\end{array}$ & 100 & daily at close \\
\hline \multirow[t]{3}{*}{ Sweden } & $\begin{array}{l}\text { JAPO Industrial } \\
\text { Banks } \\
\text { Investment }\end{array}$ & $\begin{array}{r}30 \\
9 \\
19\end{array}$ & 31 Dec 1956 & 100 & daily at close \\
\hline & Affarvarden & 135 & 31 Dec 1979 & 100 & daily at close \\
\hline & Veckans Affarer & 135 & 31 Dec 1972 & 100 & weekly \\
\hline \multirow[t]{3}{*}{ Switzerland } & Credit Suisse Index (CSI) & variable & 31 Dec 1959 & 100 & daily at close \\
\hline & $\begin{array}{l}\text { Societe Generale de Banque Suisse } \\
\text { Index (SBSI) }\end{array}$ & variable & 31 Dec 1958 & 100 & daily at close \\
\hline & $\begin{array}{l}\text { Banque Nationale Suisse Index } \\
\text { (BNSI) }\end{array}$ & variable & 1966 & 100 & $\begin{array}{l}\text { weekly } \\
\text { (Friday's } \\
\text { prices) }\end{array}$ \\
\hline \multirow[t]{2}{*}{$\begin{array}{l}\text { United } \\
\text { Kingdom }\end{array}$} & $\begin{array}{l}\text { Financial Times-Actuaries all-share } \\
\text { Index (FTA) }\end{array}$ & 750 (app.) & 10 Apr 1962 & 100 & daily at 3.30 \\
\hline & $\begin{array}{l}\text { Financial Times Industrial Ordinary } \\
\text { shares Index (FTO) }\end{array}$ & 30 & $1 \mathrm{Jul} 1935$ & 100 & hourly \\
\hline
\end{tabular}


World Stock Exchange Indices xiii

Country Index

Financial Times-Stock Exchange (FT-SE) 1000 Index

USA Amex market value index

Dow Jones Average (see text)

NYSE Composite

Standard \& Poor's

Standard \& Poor's

Standard \& Poor's

$\begin{array}{ccll}\begin{array}{c}\text { No. of } \\ \text { Companies }\end{array} & \text { Base date } & \begin{array}{l}\text { Base } \\ \text { Value }\end{array} & \begin{array}{l}\text { Frequency of } \\ \text { Calculation }\end{array} \\ 100 & 30 \text { Dec } 1983 & 1000 & \text { continuously }\end{array}$

all (860 app.) 1 Sep $1973 \quad 100$

$10: 15,10: 30$

then every

half-hour

$\begin{array}{cccc}\text { all (1560 app.) } & 31 \text { Dec } 1965 & 50 & \text { continuously } \\ 500 & 1941 / 3 & 10 & \text { continuously } \\ 400 & 1941 / 3 & 10 & \text { continuously } \\ 100 & \text { 2 Jan } 1976 & 100 & \text { continuously }\end{array}$

This table is largely based on statistics drawn up by the Brussels Stock Exchange for the F.I.B.V. in 1983. The F.I.B.V.'s permission to use it is gratefully acknowledged. 